\title{
$\mathrm{V}$ 掺杂对 Ni-Co-S/细菌纤维素基碳气凝胶 电催化性能的影响
}

\author{
吕子夜，唐谊平，曹华珍，郑国渠，侯广亚 \\ (浙江工业大学 材料科学与工程学院, 杭州 310014)
}

摘 要: 本研究以细菌纤维素基碳气凝胶 $(\mathrm{CA})$ 为载体材料, 通过水热法制备了 $\mathrm{Ni}-\mathrm{Co}-\mathrm{S} / \mathrm{CA}$ 复合气凝胶, 掺入钒元素 调控材料的微观结构和性能。研究结果表明: Ni-Co-S 的主要物相为 $\mathrm{NiCo}_{2} \mathrm{~S}_{4}$, 次相为 $\mathrm{NiS}_{2}$ 。随着镍钴盐浓度的增大, 负载量增加, 电催化峰电流密度先升后降。当镍钴盐浓度较低时, 掺微量钒后, Ni-Co-S 从结晶度较高的球形颗粒转 变成低结晶度的方形微粒, 电催化活性和稳定性都得到改善。在 $0.01 \mathrm{~mol} / \mathrm{L}$ 镍钴盐溶液中掺入 $3 \mathrm{~mol} \% \mathrm{~V}$, 制备的电 极对甲醇具有最优的催化氧化性能，与不掺 $\mathrm{V}$ 的样品相比，其峰电流密度 $\left(78.18 \mathrm{~mA} / \mathrm{cm}^{2}\right)$ 提升了至少 $45.7 \%$ 。 $\mathrm{Ni}-\mathrm{Co}-\mathrm{S} / \mathrm{CA}$ 复合气凝胶电极具有轻质、高孔隙率等优点, 有望用于便携式直接甲醇燃料电池。

关 键 词: 细菌纤维素; 碳气凝胶; 镍钴硫; 钒掺杂; 甲醇氧化

中图分类号: TQ174 文献标识码: A

\section{Effect of V Doping on Electrocatalytic Performance of Ni-Co-S on Bacterial Cellulose-derived Carbon Aerogel}

\author{
LYU Ziye, TANG Yiping, CAO Huazhen, ZHENG Guoqu, HOU Guangya
}

(College of Materials Science and Engineering, Zhejiang University of Technology, Hangzhou 310014, China)

\begin{abstract}
Ni-Co-S/CA composite aerogels were prepared by hydrothermal method using bacterial cellulose-derived carbon aerogels (CA) as support. The microstructure and properties of the composites were adjusted via adding trace vanadium. The characterization results show that the main phase of $\mathrm{Ni}-\mathrm{Co}-\mathrm{S}$ is $\mathrm{NiCo}_{2} \mathrm{~S}_{4}$ with the secondary phase of $\mathrm{NiS}_{2}$. With the increment of the nickel-cobalt salt concentration, the load amount increases, and the peak current density of electrocatalysis firstly upgraded and then degraded. After being doped with a small amount of vanadium at lower nickel-cobalt salt concentration, Ni-Co-S transforms from spherical particles with high crystallinity to square particles with low crystallinity, and its electrocatalytic activity and stability are improved. Under the preparative conditions of $0.01 \mathrm{~mol} / \mathrm{L}$ total concentration of nickel-cobalt salt and $3 \mathrm{~mol} \%$ vanadium salt, the as-obtained electrode exhibits the optimal catalytic performance for methanol oxidation. Compared with the sample without V doping, its peak current density $\left(78.1 \mathrm{~mA} / \mathrm{cm}^{2}\right)$ enhanced by $45.7 \%$ at least. The Ni-Co-S/CA composite aerogel electrodes with the advantages of light weight and high porosity, is expected to be applied in portable direct methanol fuel cell.
\end{abstract}

Key words: bacterial cellulose; carbon aerogel; Ni-Co-S; vanadium doping; methanol oxidation

收稿日期：2020-01-08; 收到修改稿日期：2020-03-04

基金项目: 国家自然科学基金(51101140); 浙江省自然科学基金(LY16E010004)

National Natural Science Foundation of China (51101140); The Natural Science Foundation of Zhejiang Province (LY16E010004)

作者简介：吕子夜(1995-), 男, 硕士研究生. E-mail: 2542990751@qq.com LYU Ziye(1995-), male, Master candidate. E-mail: 2542990751@qq.com

通讯作者：侯广亚，副教授. E-mail: hougunagya@zjut.edu.cn HOU Guangya, associate professor. E-mail: hougunagya@zjut.edu.cn 
为了寻求清洁和可持续能源, 科研人员们开展 了大量探索 ${ }^{[1-5]}$, 发现直接甲醇燃料电池(DMFC) 具 有结构简单、效率高、能量密度高、燃料储运及补 充方便等优点, 特别适合用作便携式移动电源 ${ }^{[4]}$ 。现 阶段, Pt 是 DMFC 氧化最有效的催化剂, 但其高成 本和运行过程中的 $\mathrm{CO}$ 中毒现象, 限制了 DMFC 的 大规模商业化应用 ${ }^{[6-8]}$ 。

为解决上述问题, 研究人员采用了多种措施, 如: 制备 Pt 纳米级颗粒, 提高其利用率并降低负载 量 ${ }^{[9]}$; 与其他金属形成合金以降低 $\mathrm{Pt}$ 负载量并提高 抗中毒能力 ${ }^{[10]}$; 通过金属之间的异质结构增加活性 位点 ${ }^{[1]}$; 寻求合适的载体以提高电极的导电性和颗 粒的分散性 ${ }^{[11-12] ;}$; 遴选非贵金属催化剂替代 Pt。

在各种非 Pt 基催化剂中，镍及其合金或化合物 可在碱性溶液中电催化氧化甲醇, 有望替代贵金属 成为新型电催化材料 ${ }^{[13-14]}$ 。镍的硫化物具有良好的 催化活性和抗中毒能力 ${ }^{[15-16]}$, 被用于醇类氧化催 化。Co 元素可以促进镍基催化剂对许多小分子的电 化学氧化作用 ${ }^{[17-18]}$, 并消除中间产物中毒和提高电 化学性能。因此, 镍钴基材料如 NiCoPO, Ni-B-Co, $\mathrm{NiCo} / \mathrm{NiO}-\mathrm{CoO}^{[19-21]}$ 等, 被认为是有潜力的电催化 材料。而 Ni-Co-S 三元材料用于甲醇电催化氧化的 研究鲜见文献报导。

掺杂 $\mathrm{V}$ 是改变催化剂晶体结构和提升性能的一 种重要方法 ${ }^{[22-24]}$, 如 $\mathrm{Ma}$ 等 ${ }^{[25]}$ 通过水热法制备掺 $\mathrm{V}$ 的 $\mathrm{Ni}_{3} \mathrm{~S}_{2}$ 纳米片具有特殊阵列, 可以提供高接触面 积, 进而提高催化水分解能力。

催化电极中载体材料也是影响性能的重要因 素。在负载催化剂的载体材料中, 碳基材料具有轻 质、导电和高比表面积的优点 ${ }^{[3,26]}$, 如炭黑、多孔碳、 碳纳米管、碳气凝胶、石墨烯或氮掺杂碳材料

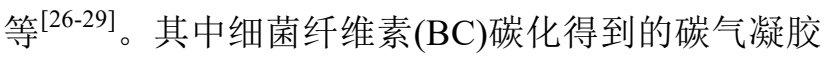
(CA)为三维网络结构 ${ }^{[30]}$, 具有柔性优异、比表面积 大和导电性好的优点。并且 $\mathrm{CA}$ 纤维上的- $\mathrm{OH}$ 可以 为活性物质的沉积提供丰富的位点。

本研究使用低成本的 $\mathrm{BC}$ 基碳气凝胶为载体材 料, 借助水热法负载非贵金属的镍钴基硫化物，制 备 Ni-Co-S/CA 复合催化电极, 通过掺 V 进一步提升催 化性能。研究镍钴盐浓度和掺 $\mathrm{V}$ 量对 $\mathrm{Ni}-\mathrm{Co}-\mathrm{S} / \mathrm{CA}$ 复 合电极的物相、显微结构及对甲醇电催化性能的影响。

\section{1 实验方法}

\section{1 原料}

细菌纤维素 $(\mathrm{BC})$ 水凝胶膜购自海南光宇生物科 技有限公司。 $\mathrm{Ni}\left(\mathrm{NO}_{3}\right)_{2} \cdot 6 \mathrm{H}_{2} \mathrm{O} 、 \mathrm{Co}\left(\mathrm{NO}_{3}\right)_{2} \cdot 6 \mathrm{H}_{2} \mathrm{O}$ 、
$\mathrm{NH}_{4} \mathrm{VO}_{3}$ 、硫腿 $\left(\mathrm{CH}_{4} \mathrm{~N}_{2} \mathrm{~S}\right)$ 购自阿拉丁试剂有限公司, 均为分析纯。

\section{2 碳气凝胶 $(\mathrm{CA})$ 的制备}

将 $\mathrm{BC}$ 膜裁成 $2 \mathrm{~cm} \times 2 \mathrm{~cm}$ 的小片, 浸泡在 $300 \mathrm{~mL} 0.2 \mathrm{~mol} / \mathrm{L} \mathrm{NaOH}$ 溶液中, $80{ }^{\circ} \mathrm{C}$ 水浴 $2 \mathrm{~h}$ 。自 然冷却后, 将 $\mathrm{NaOH}$ 溶液换成去离子水, $80{ }^{\circ} \mathrm{C}$ 水浴 $1 \mathrm{~h}$ 。重复上述步骤, 直至自然冷却后的溶液呈中性。 为更好地保持 $3 \mathrm{D}$ 空间结构, 将纯化后的 $\mathrm{BC}$ 直接放 入冷冻干燥机中干燥 $12 \mathrm{~h}$, 得到 $\mathrm{BC}$ 气凝胶。为降 低裧烧收缩率, 将 $\mathrm{BC}$ 气凝胶用碳纸包裹, 放入管 式炉, 在 $\mathrm{N}_{2}$ 气氛下以 $5{ }^{\circ} \mathrm{C} / \mathrm{min}$ 升温至 $700{ }^{\circ} \mathrm{C}$, 保温 $2 \mathrm{~h}$ 后, 随炉冷却至室温, 即得碳气凝胶 $(\mathrm{CA})$ 。

\section{$1.3 \mathrm{Ni}-\mathrm{Co}-\mathrm{S} / \mathrm{CA}$ 和掺 $\mathrm{V}$ 的 $\mathrm{Ni}-\mathrm{Co}-\mathrm{S} / \mathrm{CA}$ 的制备}

典型实验: 配置 $30 \mathrm{~mL}$ 硝酸镍、硝酸钴和硫艮 的混合水溶液, 镍钴盐总浓度为 $0.01 \sim 0.1 \mathrm{~mol} / \mathrm{L}$, 其 中镍钴盐的摩尔比为 $1: 2$, 镍钴盐与硫腿的摩尔比 为 $1: 4.5$ 。将上述溶液转移至 $100 \mathrm{~mL}$ 水热釜内胆, 加入 3 片 CA 后装入水热釜, 在 $180{ }^{\circ} \mathrm{C}$ 下水热反应 $2 \mathrm{~h}$ 。随炉冷却至室温后, 取出试样, 清洗, 直接冷冻 干燥 $6 \mathrm{~h}$, 即得到柔性 Ni-Co-S/CA。

为研究水热溶液中镍钴盐浓度对催化电极的微 观形貌与催化性能的影响, 保持其他参数不变, 镍 钴盐浓度为 $0.01 、 0.02 、 0.05$ 和 $0.1 \mathrm{~mol} / \mathrm{L}$, 制备的试 样分别标记为 NCS- $n(n=1,2,5,10)$ 。

在镍钴盐浓度为 0.01 和 $0.02 \mathrm{~mol} / \mathrm{L}$ 的溶液中加 入 $\mathrm{NH}_{4} \mathrm{VO}_{3}$, 加入的量占镍钴盐浓度的 $3 \mathrm{~mol} \%$ 、 $5 \mathrm{~mol} \%$ 和 $10 \mathrm{~mol} \%$, 其余工艺一致, 研究 $\mathrm{V}$ 的掺入量 对材料性能的影响。制备的试样分别标记为 $\mathrm{V} x$-NCS- $n$ $(x=3,5,10 ; n=1,2)$ 。

\section{4 材料表征}

采用场发射扫描电子显微镜(FESEM)和场发射 透射电子显微镜(FETEM) 对样品的形貌、尺寸、晶 面等进行表征，获取高角环形暗场像-扫描透射电 子像(HAADF-STEM), 用配套的能谱仪(EDS)进行 元素分析。采用日本 UltimaIV 型组合式多功能 $\mathrm{X}$ 射线衍射仪 (XRD) 分析物相 $(\mathrm{Cu}$ 靶, $\lambda=0.1546 \mathrm{~nm}$, $2 \theta=10^{\circ} \sim 90^{\circ}$, 扫描速度 $20\left(^{\circ}\right) / \mathrm{min}$ 。使用 Kratos AXIS Ultra DLD X 射线光电子能谱(XPS)测定样品的表面 化学状态。

\section{5 电化学性能测试}

使用上海辰华公司的 CHI760 电化学工作站测 试样品的电化学性能。分别在 $1 \mathrm{~mol} / \mathrm{L} \mathrm{KOH}$ 水溶液 以及 $1 \mathrm{~mol} / \mathrm{L} \mathrm{KOH}+0.5 \mathrm{~mol} / \mathrm{L}$ 甲醇的水溶液中, 以制 备的催化电极为工作电极, 以 $\mathrm{Pt}$ 片 $(1 \mathrm{~cm} \times 1 \mathrm{~cm})$ 为对 电极, 饱和甘录电极为参比电极 $(\mathrm{SCE}, E=0.268 \mathrm{~V}(v \mathrm{~s}$ 
$\mathrm{NHE})$ ), 以 $50 \mathrm{mV} / \mathrm{s}$ 的扫速, 在 $-0.5 \sim 1 \mathrm{~V}$ 的电位区间 进行循环伏安 $(\mathrm{CV})$ 测试。在进行 $\mathrm{CV}$ 变量测试时, 甲 醇浓度选择 $0.05 \sim 0.5 \mathrm{~mol} / \mathrm{L}$ ，扫描速率为 10 $50 \mathrm{mV} / \mathrm{s}$ 。并在 $0.8 \mathrm{~V}$ 下进行计时电流 $(I-t)$ 测试。采 用循环伏安测试 1000 次, 以表征电极的使用寿命。

\section{2 结果与讨论}

\section{1 晶体结构和元素分布}

图 1(a)是 CA、NCS-2、NCS-10 和 V3-NCS-1 的 XRD 图谱。除去载体 $\mathrm{CA}$ 和固定试样用的橡皮泥 的衍射峰, NCS- $n$ 样品中的衍射峰表明主相为 $\mathrm{NiCo}_{2} \mathrm{~S}_{4}$, 次相为 $\mathrm{NiS}_{2}$, 说明高结晶度的 $\mathrm{NiCo}_{2} \mathrm{~S}_{4}$ 和 $\mathrm{NiS}_{2}$ 共存于 $\mathrm{CA}$ 内部。掺 $\mathrm{V}$ 后试样物相发生明显变 化, Ni-Co-S 颗粒中没有明显的 $\mathrm{NiCo}_{2} \mathrm{~S}_{4}$ 衍射峰, 且 $\mathrm{NiS}_{2}$ 的衍射峰变宽, 表现出非晶峰的特征, 说明 $\mathrm{V}$ 掺杂改变了材料的结晶度。

为了进一步分析 $\mathrm{Ni}-\mathrm{Co}-\mathrm{S} / \mathrm{CA}$ 的元素组成和化 学状态, 对 NCS-2 进行 XPS 分析。XPS 全谱(图 1(b)) 表明 NCS-2 由 Ni、Co、S、C 和 O 组成, O 元素可 能来自于空气。图 1(c e e) 分别是 $N i 2 p, C o 2 p$ 和 $S 2 p$ 的高分辨率 XPS 光谱图。Ni2p 和 Co2p XPS 光谱图 可以很好地拟合出 2 个自旋轨道双峰和 2 个振动卫 星峰。对于 Ni 2p XPS 光谱图, 853.1 和 $870.1 \mathrm{eV}$ 处 的峰与 $\mathrm{Ni}^{2+}$ 有关, 855.9 和 $873.3 \mathrm{eV}$ 处的峰是 $\mathrm{Ni}^{3+}$ 的
特征峰。Co2p XPS 光谱图中, 在 778.5 和 $793.6 \mathrm{eV}$ 的峰归属于 $\mathrm{Co}^{3+}$, 并且在 781.2 和 $796.9 \mathrm{eV}$ 的峰对 应于 $\mathrm{Co}^{2+}$ 。 $\mathrm{S} 2 \mathrm{p}$ 光谱中位于 161.2 和 $162.5 \mathrm{eV}$ 的 2 个主峰, 分别对应 $S 2 p_{3 / 2}$ 和 $S 2 p_{1 / 2}$ 。 $162.5 \mathrm{eV}$ 处的峰 归属于 $\mathrm{Ni}-\mathrm{S}$ 和 $\mathrm{Co}-\mathrm{S}$ 键, 而 $161.2 \mathrm{eV}$ 处的峰归属于 表面处于低配位状态的硫离子, 这与硫空位有关 ${ }^{[31]}$ 。 上述 XPS 分析结果表明在 NCS-2 中 $\mathrm{Ni}^{2+} 、 \mathrm{Ni}^{3+} 、 \mathrm{Co}^{2+}$ 、 $\mathrm{Co}^{3+}$ 和 $\mathrm{S}^{2-}$ 共同存在。

\section{2 表面形貌}

图 2(a) 是碳气凝胶的 SEM 照片。CA 具有纳米 纤维 3D 网络结构, 孔径丰富, 比表面积大, 有利于 后续材料的制备和反应过程中物质的传输。从 NCS- $n$ 的 SEM 照片(图 2(b f)) 中可见, 颗粒负载在 CA 的纤维上。图 2(d) 为 NCS-2 中颗粒的 SEM 照片。 颗粒生长在 CA 交织的网络中, 被牢牢地 “绑在” $\mathrm{CA}$ 内部。随着镍钴盐浓度的增加, Ni-Co-S 的负载 量加大, 当镍钴盐浓度达到 $0.1 \mathrm{~mol} / \mathrm{L}$ 时(图 2(f))时, CA 内部空间中分布了大量不规则的 Ni-Co-S 小颗 粒。有趣的是, 低浓度时颗粒生长分布不均匀, 且颗 粒较大。这可能是因为在纤维交叉缠结部分或纤维 中断部分, $-\mathrm{OH}$ 较为丰富、易于形核, Ni-Co-S 在此 部分优先生长。当镍钴盐浓度增大后, Ni-Co-S 除了 在适宜部位生长外, 也可以依附在纤维其余部分异 质形核，颗粒尺寸差异减小。

图 3(a c) 为 NCS-2 的 TEM 照片和 EDS 元素分
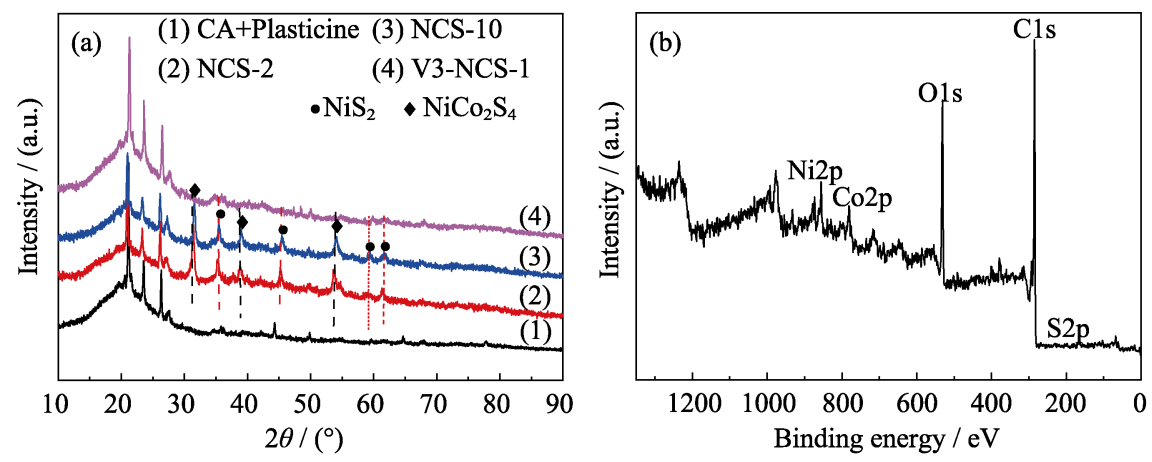

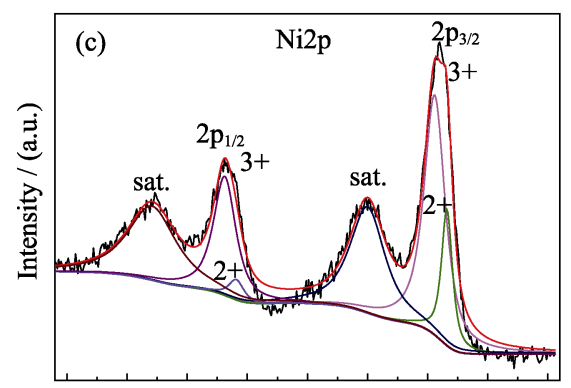

$\begin{array}{lllllllll}885 & 880 & 875 & 870 & 865 & 860 & 855 & 850 & 845\end{array}$ Binding energy / eV
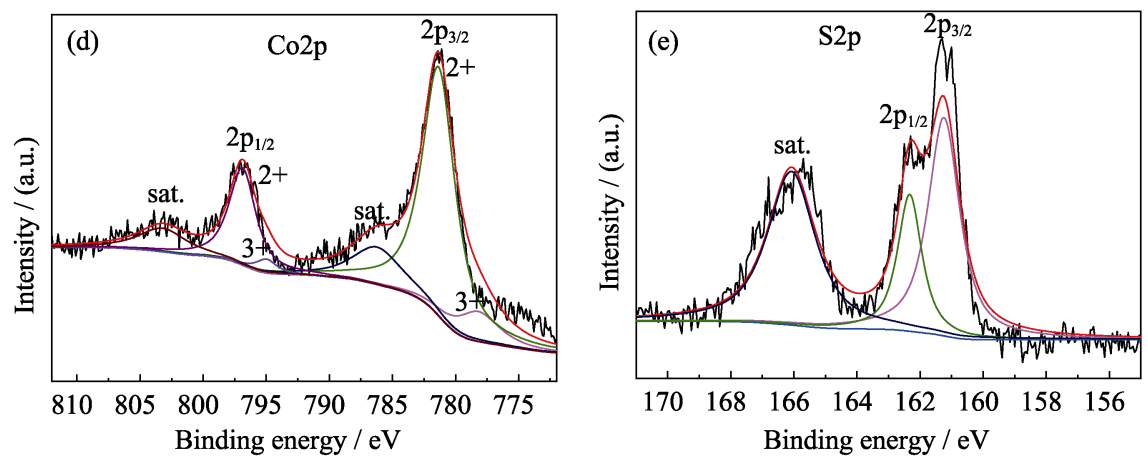

图 1 (a) CA、NCS-2、NCS-10 和 V3-NCS-1 的 XRD 图谱; NCS-2 的 XPS (b)全谱、 (c) Ni2p、(d) Co2p 和(e) S2p 谱图 Fig. 1 (a) XRD patterns of CA, NCS-2, NCS-10 and V3-NCS-1 samples; (b) Total survey, (c) Ni2p, (d) Co2p and (e) S2p XPS spectra of NCS-2 

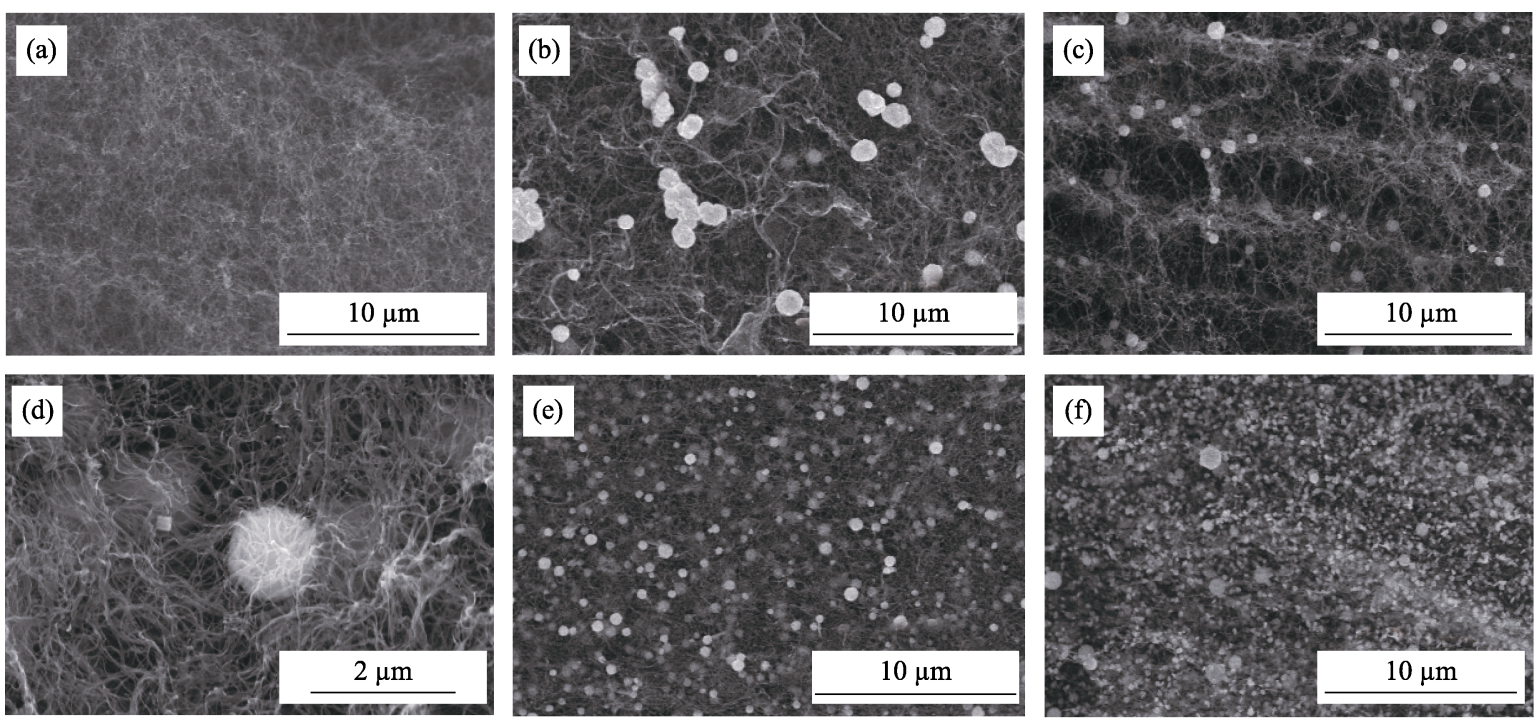

图 2 (a)碳气凝胶, (b) NCS-1, (c, d) NCS-2, (e) NCS-5 和(f) NCS-10 的 SEM 照片

Fig. 2 SEM images of (a) carbon aerogels, (b) NCS-1, (c, d) NCS-2, (e) NCS-5 and (f) NCS-10
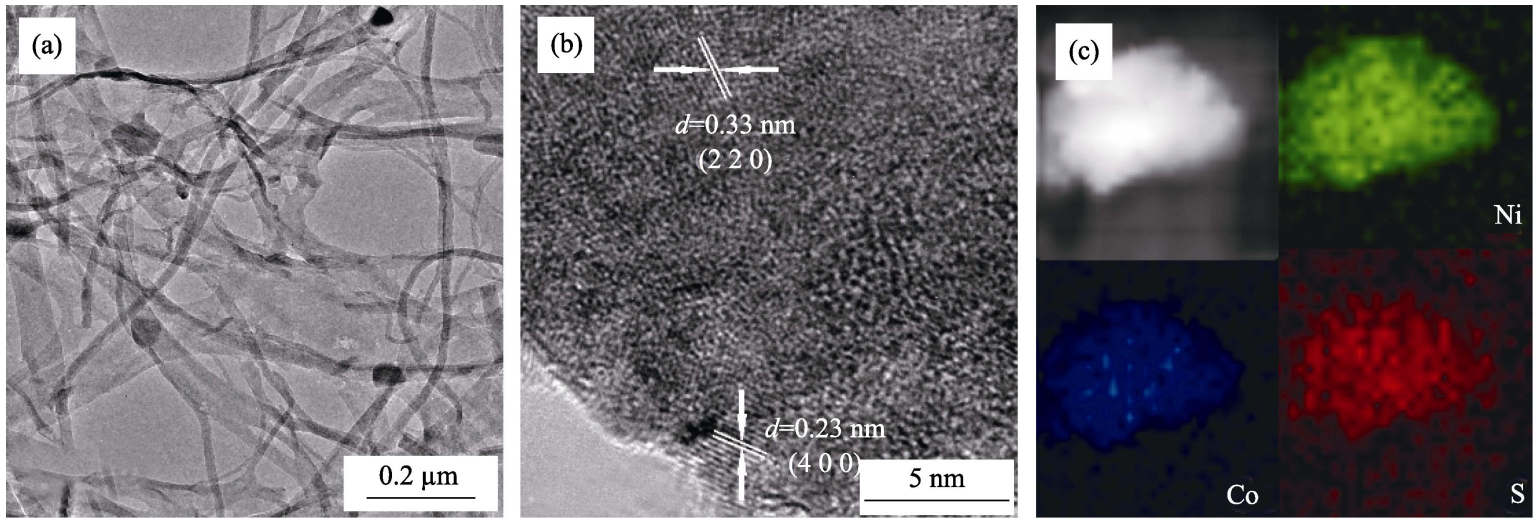

图 3 NCS-2 的(a b)高分辨 TEM 照片, (b) HAADF-STEM 照片以及相应(c) EDS 元素面分布图

Fig. 3 (a-b) High-resolution TEM image, (b) HAADF-STEM image and (c) EDS element mappings of NCS-2

布图。从图 3(a)中可观察到气凝胶的多孔网络结构, 碳纤维直径约为 $14 \mathrm{~nm}$, 颗粒依附于纤维表面或纤 维端部。图 3(b)中, NCS-2 的晶面间距为 0.23 和 $0.33 \mathrm{~nm}$, 分别对应于 $\mathrm{NiCo}_{2} \mathrm{~S}_{4}$ 的(400)和(220)晶面, 说明 Ni-Co-S 有较高的结晶度。EDS 元素分布图 (图 3(c))进一步证实 $\mathrm{Ni} 、 \mathrm{Co}$ 和 $\mathrm{S}$ 元素均匀分布在颗 粒中。

图 4(a d) 分别为 NCS-1 和 V $x$-NCS-1 $(x=3,5,10)$ 的 SEM 照片。可以看出未掺杂时, 颗粒为近球形, 表面较为光洁。当掺 V 量较少时, 颗粒形态为带正 交“沟壑”的方形，且表面较为粗糙(图 4(b))。在 V 掺 入量较大时, 沟壑逐渐消失, 向球形转变, 但结晶 度没有明显改善(图 4(c c d)), 与 XRD 分析结果一 致。当掺入微量 $\mathrm{V}$ 时，颗粒的生长方式和结晶度发 生变化, 促进立方晶型 $\mathrm{NiS}_{2}$ 的形核, 后续复合生长 了钴硫化物，从而形成具有更大比表面积、带正交 “沟壑”的方形颗粒，这与 Baig 等 ${ }^{[32]}$ 的报道相一致。
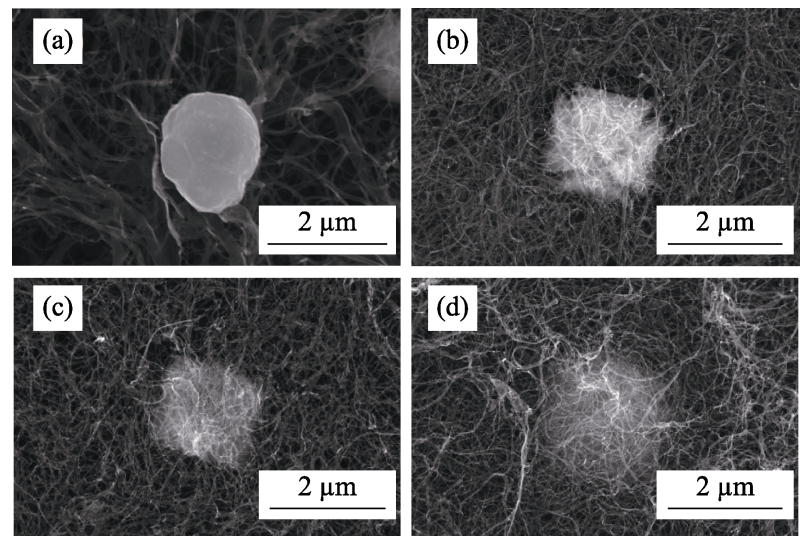

图 4 (a) NCS-1 和 $\mathrm{V} x$-NCS-1 ( $x=3(\mathrm{~b}), 5(\mathrm{c}), 10(\mathrm{~d}))$ 的 SEM 照片 Fig. 4 SEM images of (a) NCS-1 and V $x$-NCS-1 ( $x$ is (b)3, (c) 5 or (d) 10$)$

\section{3 电化学性能}

从不同镍钴盐浓度下制备的样品 CV 曲线(图 5(a)) 中可看出, 随着镍钴盐浓度上升, 在 $1 \mathrm{~mol} / \mathrm{L} \mathrm{KOH}+$ 

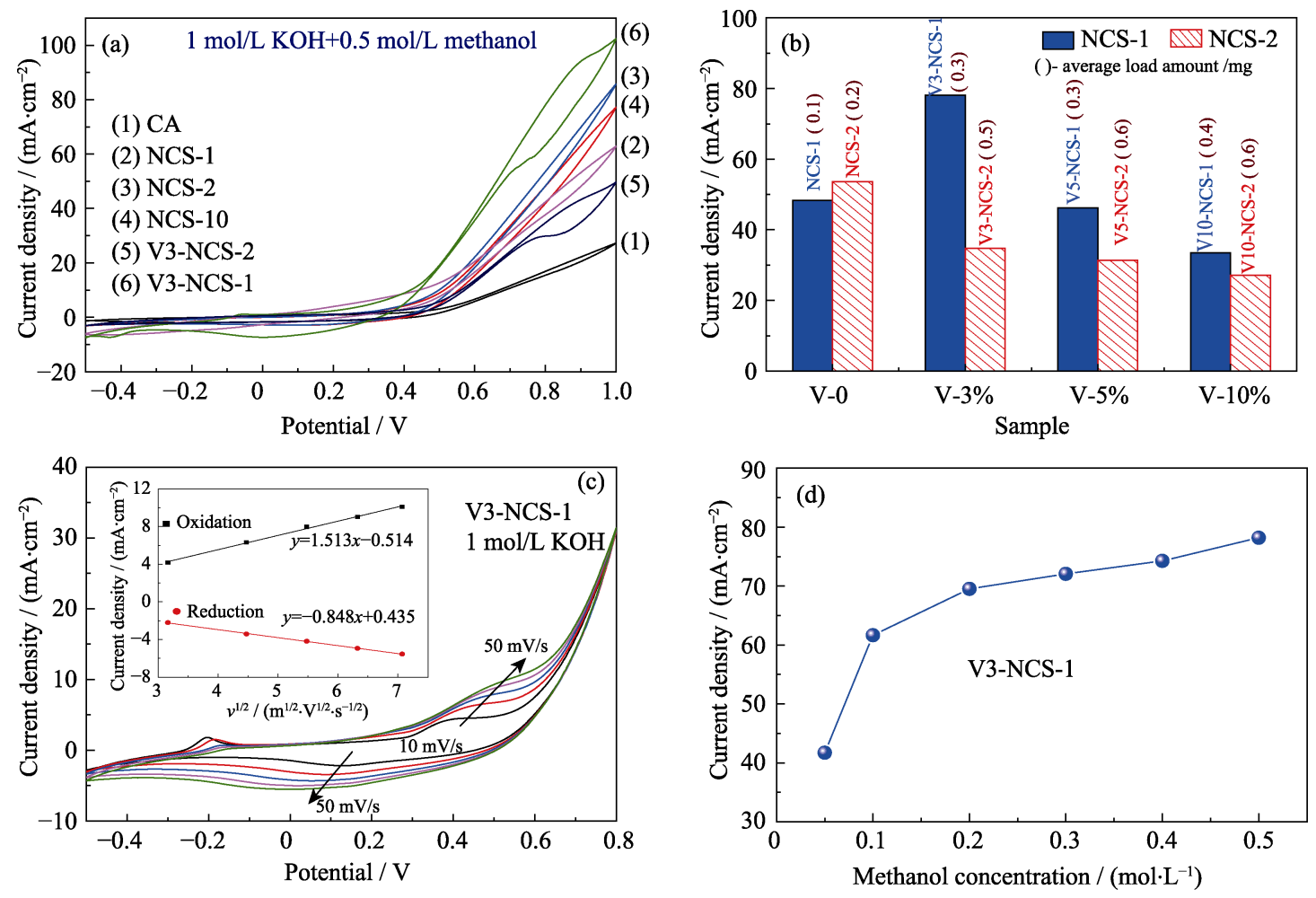

图 5 (a) CA、 NCS- $n(n=1,2,10)$ 和 V3-NCS- $n(n=1,2)$ 的 CV 曲线; (b) NCS- $n(n=1,2)$, V $x$-NCS- $n(x=3,5,10 ; n=1,2)$ 的峰值电流 密度和平均负载量对比图; (c) V3-NCS-1 在 $1 \mathrm{~mol} / \mathrm{L} \mathrm{KOH}$ 中，以 $10 、 20 、 30 、 40 、 50 \mathrm{mV} / \mathrm{s}$ 扫描的 $\mathrm{CV}$ 曲线，插图为峰值电流 密度与扫速平方根之间的关系; (d) V3-NCS-1 的峰电流密度与甲醇浓度的关系曲线

Fig. 5 (a) CV curves of CA, NCS- $n(n=1,2,5,10)$ and V3-NCS- $n(n=1,2)$; (b) Comparison of the peak current density and the average load amount of NCS- $n(n=1,2), \mathrm{V} x$-NCS- $n(x=3,5,10 ; n=1,2)$; (c) CV curves of V3-NCS-1 in 1 mol/L KOH at 10, 20, 30, $40,50 \mathrm{mV} / \mathrm{s}$ sweep speed with inset showing the relationship between the peak current density and the square root of the sweep speed; (d) Relationship between the methanol concentration and the peak current density of V3-NCS-1

$0.5 \mathrm{~mol} / \mathrm{L}$ 甲醇水溶液中电极氧化峰电流密度首先 上升, NCS-2 达到最高 $\left(53.60 \mathrm{~mA} / \mathrm{cm}^{2}\right)$, 然后下降。 当镍钴盐总浓度 $\leqslant 0.02 \mathrm{~mol} / \mathrm{L}$ 时, 颗粒均匀分布在 CA 内部，通过 CA 纤维相互贯通，有效催化面积大， 催化能力强。当镍钴盐总浓度 $\geqslant 0.05 \mathrm{~mol} / \mathrm{L}$ 时, 颗粒 逐渐填充 CA 内部, 无法发挥 CA 高电导率的优势, 颗粒长大和堆积也导致催化活性面积减小, 催化能 力下降。

从图 5(a b) 可以看出掺 V 量对 NCS-1 和 NCS-2 电化学性能的影响。对于 NCS-1, 随着掺 V 量的增 加, $0.8 \mathrm{~V}$ 处的峰电流密度先升高后降低。V3-NCS-1 的峰电流密度达到 $78.18 \mathrm{~mA} / \mathrm{cm}^{2}$, 比未掺 $\mathrm{V}$ 的最优 电极 NCS-2 高 $24.58 \mathrm{~mA} / \mathrm{cm}^{2}$, 提升了 $45.7 \%$ 。对于 NCS-2, 随着掺 V 量的增加, 峰电流密度下降。这说 明溶液中在镍钴盐含量较低时, 掺入少量 $\mathrm{V}$ 元素, 一方面改变 Ni-Co-S 的颗粒形貌, 形成有正交“沟 壑”的结晶度较低的方形颗粒, 另一方面促进了水 热过程中的形核, 一定程度上提升了 $\mathrm{CA}$ 的负载量, 有助于提高催化性能。较高的镍钴盐溶液浓度和掺 入较多的 $\mathrm{V}$ 元素都导致 $\mathrm{CA}$ 的负载量增加, 颗粒长
大并形成堆积, 还有可能导致 $\mathrm{V}$ 的硫化物或氧化物 析出, 从而使催化活性降低 ${ }^{[33]}$ 。可见, 在一定制备 条件下，掺入少量 $\mathrm{V}$, 可以在低的镍钴盐浓度下获 得较高催化性能的材料。

为进一步探讨 V3-NCS-1 的电化学行为, 在 $1 \mathrm{~mol} / \mathrm{L} \mathrm{KOH}$ 溶液中进行循环伏安测试(图 5(c)), 0.38 和 $0.13 \mathrm{~V}$ 处有一对氧化还原峰, 氧化还原反应 可用式(1 2)描述 ${ }^{[34]}$ :

$$
\begin{gathered}
\mathrm{NiCO}_{2} \mathrm{~S}_{4}+\mathrm{OH}^{-}+\mathrm{H}_{2} \mathrm{O} \rightarrow \mathrm{NiSOH}+2 \mathrm{CoSOH}+2 \mathrm{e}^{-} \\
\mathrm{CoSOH}+\mathrm{OH}^{-} \rightarrow \mathrm{CoSO}+\mathrm{H}_{2} \mathrm{O}+\mathrm{e}^{-}
\end{gathered}
$$

峰电流密度随扫描速率的增加而增大, 氧化峰 电位和还原峰电位随扫描速率的增大分别正移和负 移。峰电流密度与扫速平方根具有良好的线性关系, 表明 $\mathrm{Ni}^{2+} / \mathrm{Ni}^{3+} 、 \mathrm{Co}^{2+} / \mathrm{Co}^{3+}$ 和 $\mathrm{Co}^{3+} / \mathrm{Co}^{4+}$ 之间的转变过 程都属于扩散控制。

图 5(d)为甲醇浓度与峰电流密度的关系曲线。 随着甲醇浓度的上升, 峰电流密度也逐渐增大, 这 是在氧化甲醇的过程中 $\mathrm{Ni}^{3+} 、 \mathrm{Co}^{3+}$ 和 $\mathrm{Co}^{4+}$ 的协同作 用造成的。当甲醇浓度高于 $0.3 \mathrm{~mol} / \mathrm{L}$ 时峰电流密度 的增长趋势放缓, 这是因为高浓度下甲醇吸附达到 
饱和，所以峰电流密度没有明显变化 ${ }^{[35]}$ 。

\section{4 循环稳定性}

对 NCS-2 和 V3-NCS-1 电极连续进行 1000 次 $\mathrm{CV}$ 测试，表征其循环寿命。从图 6(a)中可见，随着 循环扫描次数的增加, 两者的峰电流密度 $(00.8 \mathrm{~V})$ 总体呈下降趋势, NCS-2 在 1000 次循环后峰电流密 度保留率为 $71 \%\left(38.06 \mathrm{~mA} / \mathrm{cm}^{2}\right)$, 而 V3-NCS-1 保留 率为 $76 \%\left(59.42 \mathrm{~mA} / \mathrm{cm}^{2}\right)$ 。5 500 次循环后因电化学 氧化, $\mathrm{NiCO}_{2} \mathrm{~S}$ 表面形成了非晶金属氧化物薄膜, 具 有相对较大的表面积，导致峰电流密度上升; 随着 循环的进行，氧化物薄膜被还原，暴露出新的硫化 物表面, 峰电流密度下降; 新的表面随之被电化学 氧化, 如此峰电流密度出现起伏的现象。在 $0.8 \mathrm{~V}$ 下 对 2 个电极进行计时电流测试(图 6(b)), 表征其在高 电位下的稳定性。从图中可观察到 $3600 \mathrm{~s}$ 后 NCS-2 的电流密度保留率为 $52 \%\left(27.87 \mathrm{~mA} / \mathrm{cm}^{2}\right), \mathrm{V} 3-\mathrm{NCS}-1$ 的电流密度保留率为 $67 \%\left(52.38 \mathrm{~mA} / \mathrm{cm}^{2}\right)$ 。而且在 $500 \mathrm{~s}$ 后, V3-NCS-1 的电流密度下降缓慢, 后续稳定 性较好。可见, 掺 $3 \mathrm{~mol} \% \mathrm{~V}$ 后电极的电催化活性和 循环寿命均得到明显改善。

对 1000 次循环扫描后的 NCS-2 和 V3-NCS-1 进行 XRD、TEM 和 EDS 表征, 研究电极循环前后 物相结构和形态的变化。图 6(c)中, NCS-2 在 1000
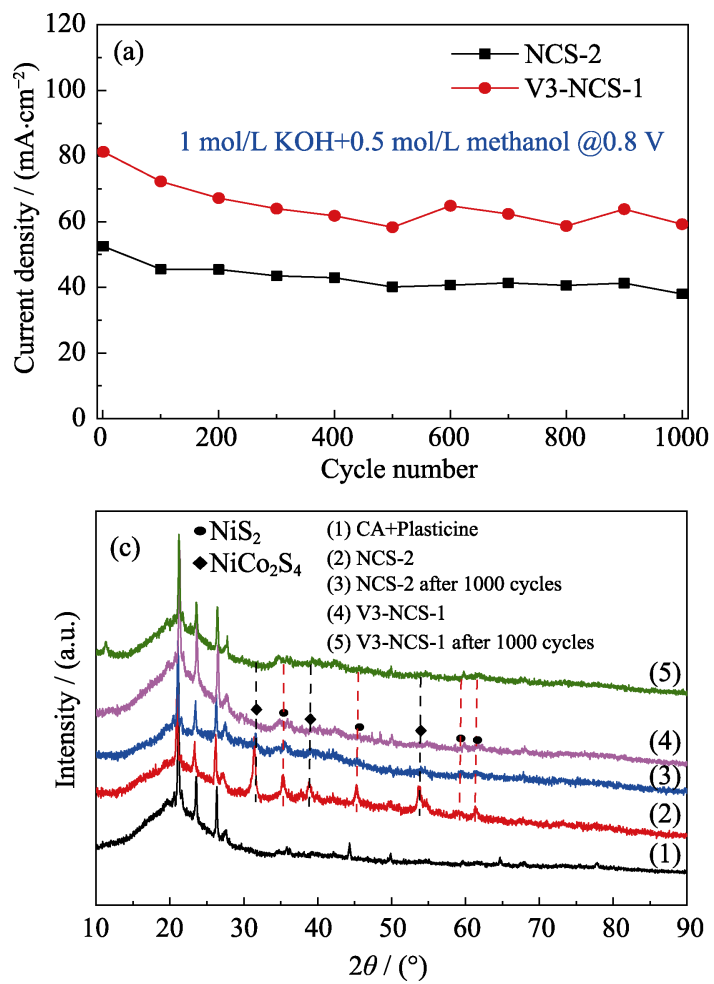

次循环扫描后，原先结晶度较高的 $\mathrm{NiCo}_{2} \mathrm{~S}_{4}$ 和 $\mathrm{NiS}_{2}$ 的衍射峰减弱, 材料的结晶度变差。而 V3-NCS-1 的物相没有明显变化, 循环扫描前后结晶度都不高, 为非晶态结构。在图 6(d)中未观察到 NCS-2 上所负 载颗粒的晶格条纹, 表示其循环扫描后转变为无定 型结构。从图 6(e)的元素分布图中可看到, $\mathrm{Ni} 、 \mathrm{Co}$ 和 $\mathrm{O}$ 元素均匀分布在颗粒上, 没有观察到 $\mathrm{S}$ 元素。 综上, $\mathrm{NiCo}_{2} \mathrm{~S}_{4}$ 经 1000 次循环后被氧化, 转变成无定 型结构的镍钴氧化物, 与 Mabayoje 等 ${ }^{[36]}$ 的报道相 一致。

\section{3 结论}

本研究以细菌纤维素基碳气凝胶(CA)为载体, 借助水热法成功制备了 $\mathrm{Ni}-\mathrm{Co}-\mathrm{S} / \mathrm{CA}$ 复合气凝胶。该 材料具有质轻, 制备过程简单, 成本低廉等优点。在 镍钴盐浓度为 $0.02 \mathrm{~mol} / \mathrm{L}$ 时, 制备得到球形的 $\mathrm{Ni}-\mathrm{Co}-\mathrm{S}$ 颗粒较均匀地分布在 CA 内部。峰电流密度达 到 $53.60 \mathrm{~mA} / \mathrm{cm}^{2}$, 并在 1000 次循环后保留率为 $71 \%$ 。

随着镍钴盐浓度的增加, 试样负载量增大, 电 催化峰电流密度先升后降。在低镍钴盐浓度下, 掺 入微量 $\mathrm{V}$, 可以改变 Ni-Co-S 微观结构、增加载体的 负载量，进而改善催化性能。掺入 $3 \mathrm{~mol} \% \mathrm{~V}$ 的
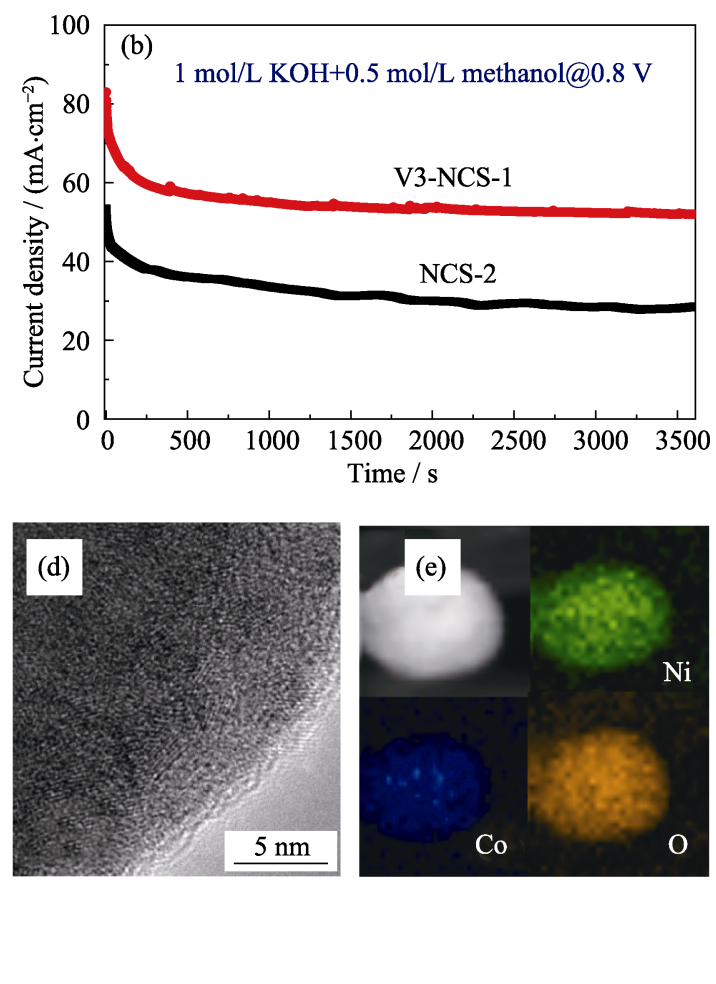

图 6 NCS-2 和 V3-NCS-1 电极循环 1000 次的(a)氧化峰电流密度变化曲线、(b)计时电流测试曲线和(c)循环前后的 XRD 图谱; NCS-2 试样中负载的颗粒 1000 次循环后的(d) TEM 照片, (e) HAADF-STEM 照片以及相应的 EDS 元素分布图

Fig. 6 (a) Oxidation peak current densities and (b) I-t curves of NCS-2 and V3-NCS-1 electrodes varied within $1000 \mathrm{CV}$ cycles, and (c) XRD patterns of NCS-2 and V3-NCS-1 before and after 1000 cycles; (d) TEM image, (e) HAADF-STEM image and corresponding EDS element mappings of the particles loaded in NCS-2 after 1000 cycles 
$\mathrm{Ni}-\mathrm{Co}-\mathrm{S} / \mathrm{CA}$ 电极的峰电流密度达到 $78.18 \mathrm{~mA} / \mathrm{cm}^{2}$, 在 1000 次 $\mathrm{CV}$ 循环后保留率达到 $76 \%$ 。掺入 $\mathrm{V}$ 为降 低电极成本提供了一条可行思路。该电极有望用于 便携式电源或微型甲醇燃料电池阳极。

\section{参考文献:}

[1] GONG S, JIANG Z, SHI P, et al. Noble-metal-free heterostructure for efficient hydrogen evolution in visible region: molybdenum nitride/ ultrathin graphitic carbon nitride. Applied Catalysis B: Environmental, 2018, 238: 318-327.

[2] LIU J, HE T, WANG Q, et al. Confining ultrasmall bimetallic alloys in porous $\mathrm{N}$-carbon for use as scalable and sustainable electrocatalysts for rechargeable $\mathrm{Zn}$-air batteries. Journal of Materials Chemistry A, 2019, 7(20): 12451-12456.

[3] LIAO K, CHEN S, WEI H, et al. Micropores of pure nanographite spheres for long cycle life and high-rate lithium-sulfur batteries. Journal of Materials Chemistry A, 2018, 6(45): 23062-23070.

[4] ZHAI C Y, DU Y K, ZHU M S. Noble metal/semiconductor photoactivated electrodes for direct methanol fuel cell. Journal of Inorganic Materials, 2017, 32(9): 897-903.

[5] LEI Y, WANG Y, LIU Y, et al. Realizing the atomic active center for hydrogen evolution electrocatalysts. Angewandte Chemie International Edition, 2020, in press, doi: 10.1002/anie.201914647.

[6] MANSOR M, TIMMIATI S N, LIM K L, et al. Recent progress of anode catalysts and their support materials for methanol electrooxidation reaction. International Journal of Hydrogen Energy, 2019, 44(29): 14744-14769.

[7] KOENIGSMANN C, WONG S S. One-dimensional noble metal electrocatalysts: a promising structural paradigm for direct methanol fuel cells. Energy \& Environmental Science, 2011, 4(4): 1161-1176.

[8] TIWARI J N, TIWARI R N, SINGH G, et al. Recent progress in the development of anode and cathode catalysts for direct methanol fuel cells. Nano Energy, 2013, 2(5): 553-578.

[9] LI C, MALGRAS V, ALSHEHRI SM, et al. Electrochemical synthesis of mesoporous Pt nanowires with highly electrocatalytic activity toward methanol oxidation reaction. Electrochimica Acta, 2015, 183: 107-111.

[10] ZHENG Y, ZHAN H, TANG H, et al. Atomic platinum layer coated titanium copper nitride supported on carbon nanotubes for the methanol oxidation reaction. Electrochimica Acta, 2017, 248: 349-355.

[11] CHEN D, HE Z, PEI S, et al. Pd nanoparticles supported on N and $\mathrm{P}$ dual-doped graphene as an excellent composite catalyst for methanol electro-oxidation. Journal of Alloys and Compounds, 2019, 785: 781-788.

[12] LUO S, GU R, SHI P, et al. $\pi$ - $\pi$ interaction boosts catalytic oxygen evolution by self-supporting metal-organic frameworks. Journal of Power Sources, 2020, 448: 227406-1-10.

[13] GUO X, LIANG T, ZHANG D, et al. Facile fabrication of 3D porous nickel networks for electro-oxidation of methanol and ethanol in alkaline medium. Materials Chemistry and Physics, 2019, 221: 390-396.

[14] LI J, LUO F, ZHAO Q, et al. Crystalline nickel boride nanoparticle agglomerates for enhanced electrocatalytic methanol oxidation. International Journal of Hydrogen Energy, 2019, 44(41): 23074-23080.

[15] ZHANG J, XU C, ZHANG D, et al. Facile synthesis of a nickel sulfide (NiS) hierarchical flower for the electrochemical oxidation of $\mathrm{H}_{2} \mathrm{O}_{2}$ and the methanol oxidation reaction (MOR). Journal of the Electrochemical Society, 2017, 164: B92-B96.

[16] YUAN G, NIU X, CHEN Z, et al. Self-supported hierarchical Shell@Core $\mathrm{Ni}_{3} \mathrm{~S}_{2} @ \mathrm{Ni}$ foam composite electrocatalyst with high efficiency and long-term stability for methanol oxidation. ChemElectro Chem, 2018, 5(17): 2376-2382.
[17] CHEN Z, WANG Q, ZHANG X, et al. N-doped defective carbon with trace Co for efficient rechargeable liquid electrolyte-/all- solid-state Zn-air batteries. Science Bulletin, 2018, 63(9): 548-555.

[18] WANG Q, YE K, XU L, et al. Carbon nanotube-encapsulated cobalt for oxygen reduction: integration of space confinement and N-doping. Chemical Communications, 2019, 55(98): 14801-14804.

[19] YANG J H, SONG X, ZHAO X, et al. Nickel phosphate materials regulated by doping cobalt for urea and methanol electro-oxidation. International Journal of Hydrogen Energy, 2019, 44(31): 16305-16314.

[20] WU F, ZHANG Z, ZHANG F, et al. Exploring the role of cobalt in promoting the electroactivity of amorphous Ni-B nanoparticles toward methanol oxidation. Electrochimica Acta, 2018, 287: 115-123.

[21] REZAEE S, SHAHROKHIAN S. Facile synthesis of petal-like $\mathrm{NiCo} / \mathrm{NiO}-\mathrm{CoO} /$ nanoporous carbon composite based on mixedmetallic MOFs and their application for electrocatalytic oxidation of methanol. Applied Catalysis B: Environmental, 2019, 244: 802-813.

[22] JIA J Y, ZHANG W X, CHANG L, et al. Synthesis and photocatalytic activity of vanadium doped titania hollow microspheres. Journal of Inorganic Materials, 2009, 24(4): 671-674.

[23] YAN Y, LI B, GUO W, et al. Vanadium based materials as electrode materials for high performance supercapacitors. Journal of Power Sources, 2016, 329: 148-169.

[24] XU X, XIONG F, MENG J, et al. Vanadium-based nanomaterials: a promising family for emerging metal-ion batteries. Advanced Functional Materials, 2020, 30(10): 1904398-1-36.

[25] MA L, ZHANG K, WANG S, et al. Vanadium doping over $\mathrm{Ni}_{3} \mathrm{~S}_{2}$ nanosheet array for improved overall water splitting. Applied Surface Science, 2019, 489: 815-823.

[26] LEI C, ZHOU W, FENG Q, et al. Charge engineering of $\mathrm{Mo}_{2} \mathrm{C} @$ defect-rich N-doped carbon nanosheets for efficient electrocatalytic $\mathrm{H}_{2}$ evolution. Nano-Micro Letters, 2019, 11(1): 45.

[27] CALVILlO L, GANGERI M, PERATHONER S, et al. Synthesis and performance of platinum supported on ordered mesoporous carbons as catalyst for PEM fuel cells: effect of the surface chemistry of the support. International Journal of Hydrogen Energy, 2011, 36(16): 9805-9814.

[28] SHI M, ZHANG W, LI Y, et al. Tungsten carbide-reduced graphene oxide intercalation compound as co-catalyst for methanol oxidation. Chinese Journal of Catalysis, 2016, 37(11): 1851-1859.

[29] WANG Q, LEI Y, ZHU Y, et al. Edge defect engineering of nitrogendoped carbon for oxygen electrocatalysts in $\mathrm{Zn}$-Air batteries. $A C S$ Applied Materials \& Interfaces, 2018, 10(35): 29448-29456.

[30] MA L N, ZHAO N, BI Z J, et al. Bacterial cellulose based nano-biomaterials for energy storage applications. Journal of Inorganic Materials, 2019, 35(2): 145-157.

[31] WANG Y, LIU Y, XIA K, et al. $\mathrm{NiCo}_{2} \mathrm{~S}_{4}$ nanoparticles anchoring on polypyrrole nanotubes for high-performance supercapacitor electrodes. Journal of Electroanalytical Chemistry, 2019, 840: $242-248$.

[32] BAIG F, HAMEED KHATTAK Y, JEMAI S, et al. Hydrothermal syntheses of vanadium doped $\alpha-\mathrm{Fe}_{2} \mathrm{O}_{3}$ cubic particles with enhanced photoelectrochemical activity. Solar Energy, 2019, 182: 332-339.

[33] ZHOU Y, LIU P, JIANG F, et al. Vanadium sulfide sub-microspheres: a new near-infrared-driven photocatalyst. Journal of Colloid and Interface Science, 2017, 498: 442-448.

[34] ZHANG H, LI H, SUN Z, et al. One-step hydrothermal synthesis of $\mathrm{NiCo}_{2} \mathrm{~S}_{4}$ nanoplates/nitrogen-doped mesoporous carbon composites as advanced electrodes for asymmetric supercapacitors. Journal of Power Sources, 2019, 439: 227082-1-9.

[35] HOU G Y, XIE Y Y, WU L K, et al. Electrocatalytic performance of Ni-Ti-O nanotube arrays/NiTi alloy electrode annealed under $\mathrm{H}_{2}$ atmosphere for electro-oxidation of methanol. International Journal of Hydrogen Energy, 2016, 41(22): 9295-9302.

[36] MABAYOJE O, SHOOLA A, WYGANT B R, et al. The role of anions in metal chalcogenide oxygen evolution catalysis: electrodeposited thin films of nickel sulfide as "pre-catalysts". ACS Energy Letters, 2016, 1(1): 195-201. 